\title{
Influencia de las dimensiones de las ramas de árboles forrajeros en el aprovechamiento por rumiantes
}

\author{
Ángel Arturo Santana-Pérez ${ }^{1}$ \\ Luis Miguel Borrás-Sandoval ${ }^{2}$ \\ Long Cheng ${ }^{3}$ \\ Danis Manuel Verdecia-Acosta ${ }^{4}$ \\ Jesús Manuel Iglesias-Gómez ${ }^{5}$ \\ Ana Mercedes Vega-Albi ${ }^{6}$ \\ Jorge Luis Ramírez-de-la-Ribera ${ }^{7}$
}

Fecha de recepción: 12 de diciembre de 2018

Fecha de aceptación: 11 de febrero de 2019

\section{Resumen}

Con el fin de determinar la longitud y el diámetro máximos hasta que son consumidos los tallos de especies arbustivas (Leucaena leucocephala, LL; Albizia lebbeck, AL; Gliricidia sepium, GS; Moringa oleifera, MO; Samanea saman, SS) por bovinos y ovinos (jóvenes y adultos), se suministraron follajes a los animales y se midieron los diámetros mínimos de las porciones de las ramas desechadas por estos (máximos diámetros de las porciones consumidas). Se parearon más de 300 mediciones de longitud/diámetro de los tallos. Se realizó un análisis de varianza triple "especie del forraje $x$ especie de animal $x$ edad del animal" (5x2x2), un análisis de regresión simple entre la longitud del tallo y su diámetro y una prueba de $Z$, para una pérdida máxima de $5 \%$ del follaje por los animales. Existieron diferencias entre los diámetros máximos de los tallos consumidos, siendo el orden general GS $>$ LL $>M O>S S>A L$, por tanto, cada especie debe ser cortada en dimensiones diferentes. También se observó que los ovinos no consumen los tallos de SS y GS. Se aconseja que el corte no sea igual para bovinos y ovinos, pero se puede ignorar la edad de estos. Además se recomienda tener en cuenta los valores exactos de las dimensiones de los tallos para cortar las especies para cada especie de animal y que se emplee esta misma metodología para evaluar otras especies de forrajes.

Palabras clave: consumo; follaje; medidas; tallos.

\footnotetext{
${ }^{1}$ Ph.D. Universidad de Granma (Bayamo-Granma, Cuba). santana@udg.co.cu. ORCID: 0000-0002-3503-9162.

2 Ph.D. Universidad Pedagógica y Tecnológica de Colombia (Tunja-Boyacá, Colombia). luis.borras@uptc.edu.co. ORCID: 0000-0002-3284-027X.

${ }^{3}$ Ph.D. University of Melbourne (Dookie, Australia). long.cheng@unimelb.edu.au.

${ }^{4}$ Ph.D. Universidad de Granma (Bayamo-Granma, Cuba). dverdeciaa@udg.co.cu. ORCID: 0000-0002-4505-4438.

${ }^{5}$ Ph.D. Estación Experimental de Pastos y Forrajes "Indio Hatuey" (Matanzas, Cuba). iglesias@ihatuey.cu. ORCID: 00000002-9501-1938.

${ }^{6}$ Ph.D. Universidad de Granma (Bayamo-Granma, Cuba). avegaa@udg.co.cu.

${ }^{7}$ Ph.D. Universidad de Granma (Bayamo-Granma, Cuba). jramirezrivera@udg.co.cu.
} 


\title{
Effects of branch dimensions of fodder trees on their utilization by ruminants
}

\begin{abstract}
To determine the stem measurements (length and diameter) up to which forage trees (Leucaena leucocephala, LL; Albizia lebbeck, AL; Gliricidia sepium, GS; Moringa oleifera, MO; Samanea saman, SS) are consumed by cattle and sheep (young or adult), these foliages were daily offered to the animals and the minimal diameters of the refused branches were measured (maximal diameter of the eaten fragment). More than 300 dimensions of stem length vs. its corresponding diameter were paired. It was done a triple analysis of variance $(5 \times 2 \times 2)$ being the factors: forage species, animal species and animal age. A simple regression analysis was done between length and diameter of the stalks, as well as a probability test of $Z$ to consider $5 \%$ refuse of the foliage by the animals. The results showed that the diameters of the eaten stems are different for each foliage species, being $G S>L L>M O>S S>A L$, this means that each one has to be cut at a different dimension, this cut must be different for cattle and sheep, but not between ages of them and that sheep do not consume any stem of SS and GS. Finally, it is recommended the exact dimensions of the stems (diameter and length) to cut each species and the evaluation methodology to be used for other fodder evaluations.
\end{abstract}

Keywords: foliage; intake; measurements; stem.

Para citar este artículo:

Santana-Pérez, A. A., Borrás-Sandoval, L. M., Cheng, L., Verdecia-Acosta, D. M., IglesiasGómez, J. M., Vega-Albi, A. M., \& Ramírez-de-la-Ribera, J. L. (2019). Influencia de las dimensiones de las ramas de árboles forrajeros en el aprovechamiento por rumiantes. Ciencia y Agricultura, 16(2), 25-38. https://doi.org/10.19053/01228420.v16.n2.2019.9118.

Esta obra está bajo licencia internacional Creative Commons Reconocimiento 4.0

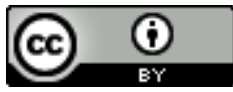




\section{INTRODUCCIÓN}

En el trópico, ante el incremento de sistemas de alimentación donde se incluyen plantas arbustivas potencialmente útiles para el ramoneo y los animales logran una tasa de consumo instantáneo mayor mediante la selección de un material con mayor acceso a las hojas y optimizando el tiempo (Wilson \& Kerley, 2003; Sebata \& Ndlovu, 2010; Sebata, 2013), es evidente que las características anatómicas de las plantas, por sus implicaciones químicas y físicas, poseen notable influencia en el aprovechamiento de estas por los animales (Dziba, Scogings, Gordon \& Raats, 2003; Papachristou, Dziba \& Provenza, 2005).

$\mathrm{Si}$, además, los tallos de las diversas plantas arbóreas forrajeras poseen elevados contenidos de fibra responsable de su rigidez (Valenciaga \& Chongo, 2004) e inversamente ligada al consumo de alimentos (Forbes, 2000; Karsli \& Russell, 2002) y también, según Laca, Shipley y Reid (2001) e Ingram et al. (2013), existen muchas interacciones entre otros elementos morfológicos de las plantas, que se denominan "características anticalidad", así como del animal, del manejo de ambos y de las condiciones ambientales, entonces, determinar cuánto es consumido de una planta arbórea por los animales en el pastoreo es un procedimiento imprescindible y complejo (Bonnet et al., 2015), lo que sugiere la necesidad de investigaciones que generen tales informaciones para el manejo sostenible de la vegetación y que puedan ser aplicadas para adoptar estrategias de suplementación (González-Pech, Torres-Acosta \& Sandoval-Castro, 2014). Se sabe, además, que los rumiantes pueden aprender de experiencias acumuladas para seleccionar los forrajes que consumen (Fisher, 2002).

La ganadería tropical urge el uso cada vez más óptimo de todos sus recursos por parte de los profesionales y la implementación de razonamientos, metodologías e instrumentos matemáticos aplicados en las ciencias biológicas, lo que ha de transformarla en las próximas décadas (NRC, 2005; Santana, Pernía \& Santana, 2015). Pero, aún en la actualidad, quienes alimentan a los animales en entidades productivas incluyen y pesan porciones significativas de follaje que luego son rechazadas.

En centros de investigaciones científicas todavía se toman muestras de follajes para sus análisis de laboratorios y pruebas con animales usando criterios imprecisos por su subjetividad, como cortar "manualmente el follaje de arbóreas considerado como tierno y comestible por los animales" (Kapinga, Bitende \& Mulangila, 1993; Aregheore, 2003; García \& Medina, 2005; Pontes, Agreil, Magda, Gleizes \& Fritz, 2010; Hernández \& Sánchez, 2014), o "las hojas y fracciones comestibles de los tallos de 30-40 cm de largo" (González-García et al., 2008) o "simulando el ramoneo que realizan los animales" (Iglesias, Matías \& Pérez, 2003; Agreil, Fritz \& Meuret, 2005; Parissi \& Nastis, 2007). Todo esto (Geisert, 2007) resume que obtener muestras precisas de alimentos consumidos por los animales pastantes es un reto muy grande, especialmente en aquellos donde lo hacen en praderas mixtas y donde 
los animales pueden cosechar diferentes partes de las plantas. Incluso se debate aún cuál método resulta mejor.

Por todas las razones anteriores y bajo la hipótesis de que se podrían encontrar notables diferencias en el nivel de consumo que hacen los bovinos y los ovinos con diferentes edades de distintas especies de árboles forrajeros, en la presente investigación se pretendió determinar la longitud y el diámetro máximos de los tallos de cinco especies de forrajes arbustivos consumidos por estos animales, que permitan recomendar las dimensiones a las que deben ser cortados sus follajes.

\section{MATERIALES Y MÉTODOS}

El experimento se realizó en las instalaciones productivas e investigativas de la Universidad de Granma, Cuba (altura de 112.5 msnm; latitud: 20ㅡ, 16',52.596" Norte; longitud: $76^{\circ}, 43^{\prime}, 36.192^{\prime \prime}$ Oeste) y donde se suministraron diariamente follajes de cinco especies de arbustivas, cortadas manualmente, a bovinos y ovinos en pastoreo divididos en dos grupos etarios.

Las cinco especies fueron:

- Albizia lebbeck (AL): durante seis días de adaptación y tres de prueba.

- Gliricidia sepium (GS): durante seis días de adaptación y cinco de prueba.

- Leucaena leucocephala (LL): durante dos días de adaptación y tres de prueba.

- Moringa oleifera (MO): durante siete días de adaptación y cinco de prueba.

- Samanea saman (SS): durante seis días de adaptación y tres de prueba.

Las plantas evaluadas estaban establecidas previamente por tres a cuatro años en un suelo pardo tropical sin carbonatos (según el Instituto Cubano de Suelos), o "Eutric Cambisoil" según la clasificación de la FAO-UNESCO (1988), que posee un contenido de materia orgánica de $3.3 \%$ y pH de neutro a ligeramente ácido $(6.7 \mathrm{a}$ 7.0), sin riego ni fertilización.

Los bovinos y ovinos pastaban en un área de cinco cuartones, manejados rotacionalmente entre siete y diez días de ocupación, en pastoreo combinado (ambas especies juntas) y cubiertos de pastos naturales (85\% de Dichantium annulatum $+D$. caricosum, $15 \%$ de otras especies no pratenses). El horario de pastoreo era de 08:00-11:30 horas y de 13:30-16:30 horas. El resto del tiempo los animales eran separados por especie (bovinos, B; ovinos, O) y edad (jóvenes, JOV; adultos, $\mathrm{AD}$ ) estabulados en cubículos únicos y con agua ad libitum.

Se establecieron cuatro grupos experimentales de animales homogéneos en edad y pesos:

1. B-JOV: cinco terneros destetados de la raza cebú, de seis $( \pm 0.5)$ meses de edad y $79( \pm 8.5) \mathrm{kg}$ de peso estimado por su perímetro torácico. 
2. B-AD: seis vacas secas y vacías de la raza cebú, con más de tres partos y peso vivo de $420( \pm 30.0) \mathrm{kg}$ de peso estimado por su perímetro torácico.

3. O- JOV: ocho machos en desarrollo de la raza pelibuey y peso vivo de 11.7 $( \pm 2.0) \mathrm{kg}$, medido con una balanza de brazo.

4. O-AD: seis reproductoras vacías de la raza pelibuey y peso vivo de 27.5 ( \pm 4.0) $\mathrm{kg}$, medido con una balanza de brazo.

Durante la estabulación diaria se les ofreció el follaje íntegro (sin trocear) y cortado manualmente, de manera tal que las ramas principales tuvieran un mínimo de 20 $\mathrm{mm}$ de diámetro en dos raciones diarias: una durante la estabulación del mediodía y la otra en la estabulación de la tarde-noche. Se le brindó a cada grupo/día una cantidad de follaje cuyo aporte de materia seca fuera equivalente al $25 \%$ del consumo requerido, según ACPA-SOCUPER (2002) para los ovinos, y según García y Cáceres (1984) para los bovinos, de tal forma que ni la cantidad ni calidad del follaje ni del pasto base limitaran el consumo del primero. El consumo de MS empleado fue del 4.5 del peso vivo (PV) para O- JOV, de $4.0 \%$ para O-AD, de 3.5 $\%$ para B- JOV y de $3.0 \%$ para B-AD.

Se midió diariamente el diámetro mínimo en el extremo de los tallos rechazados por los animales, considerado como el máximo consumido, a las 14:00 horas, de los follajes ofrecidos en la estabulación de mediodía y a las 09:00 horas en los restos del material ofrecido en la tarde-noche del día anterior. Para ello se empleó un micrómetro digital Starrett con diapasón de 0-25 mm (serie 733MEXFL-25, precisión $\pm 0.003 \mathrm{~mm}$ ). También se midió la longitud de los tallos empleando una regla graduada.

Se utilizó un método de comparación por grupos (cuatro: B- JOV, B-AD, O- JOV, O$A D$ ) y periodos de suministro de los forrajes (AL, GS, LL, MO, SS) durante un tiempo de adaptación hasta que se consideró que los animales ya consumían con avidez el follaje, y otro adicional de toma de muestras que permitiera realizar más de 100 mediciones; todo bajo un diseño trifactorial $2 \times 2 \times 5$ con la especie de animales $(B, O)$, la edad de estos (JOV, AD) y especie del forraje (AL, GS, LL, MO, SS) como factores.

El modelo matemático empleado fue:

$$
Y_{i j k l m}=\mu+F_{i}+A_{j}+E_{k}+(F \times A)_{i j}+(F \times E)_{i k}+(A \times E)_{j k}+(F \times A \times E)_{i j k}+\varepsilon_{i j k l m}
$$

Donde: $\mu$ es la media de la población, $F$ es el efecto de la i-ésima especie de forraje (1-5), $A$ es el efecto de la j-ésima especie de animal (1-2), $E$ es el efecto de la késima edad del animal (1-2) y $\mathcal{E}$ es el error residual.

Al mismo tiempo, considerando las características morfológicas de cada especie, se midieron, en cortes espaciados cada 6-8 cm, longitudes desde el extremo apical de la rama y sus respectivos diámetros de tallos en ramas secundarias y terciarias aleatorias de LL, SS y AL por ramificarse más; así como en ramas primarias de MO 
y GS que poseen tallos poco ramificados, y a partir de esto se elaboró una base con 300-325 pares de datos longitud versus diámetro.

Se realizaron análisis de varianza triple (ANOVA), de diferenciación de medias (Tukey) y de regresión que consideraron siempre significativo todo valor de $\mathrm{P}<0.05$, empleando el paquete estadístico Statitica Windows ${ }^{\mathrm{TM}}$ (StatSoft, 2011).

Para la elección de las ecuaciones de regresión cuadráticas $\left(Y=a+b X+c X^{2}\right)$ se consideró que el nivel de significación de su coeficiente $R$ fuera mayor en 0.05 unidades al menos con respecto a la lineal $(Y=a+b X)$ y que los residuales estuvieran normalmente distribuidos.

El cálculo del máximo diámetro tolerable de los tallos de cada especie de forraje, y por especie de animal, se hizo para un de $5 \%$ de pérdidas del follaje, considerando que pueden variar desde 2.5 a 8.6 \% (Flores, Amor, Resch \& González, 2000), lo que corresponde a 1.65 unidades de desviación estándar $(Z)$ según la curva de distribución teórica de probabilidades de Gauss.

\section{Resultados Y DISCUSIÓN}

En la Tabla 1 se muestran los diámetros máximos hasta los cuales fueron consumidos los tallos de las cinco especies evaluadas en cada uno de los grupos de animales y puede notarse que es mayor el consumo de LL, GS, SS y AL por bovinos que el que hacen los ovinos, pero ambas especies de animales consumieron por igual la moringa.

Los tallos de LL, GS y MO son consumidos hasta un diámetro mayor, con diferencias entre ellos, por los bovinos que los de SS y AL, que no difieren entre sí. A diferencia de esto, los ovinos consumen más LL y MO, en segundo lugar, AL, pero GS y SS no son consumidas en sus tallos. Este último aspecto no es coincidente con algunos autores (Mathews \& Ellinbank, 1989) que han hecho trabajos similares al presente, en el sentido de que no hallaron diferencias en los consumos por los bovinos y los ovinos; pero la regla muestra que sí hay diferencias entre el aprovechamiento de los tallos por ambas especies de animales. Basta analizar que, con la misma planta de tagasaste (Chamaecytisus proliferus ssp. palmensis) de los autores anteriores, en otro estudio hecho por Avendaño, Ovalle y Ramírez (2003) se registra que los bovinos consumen los tallos hasta un diámetro de $6.0 \mathrm{~mm}$, mientras que los ovinos solamente hasta $4.9 \mathrm{~mm}$ y ambos valores son muy superiores a todos los medidos en las cinco especies evaluadas en la presente investigación. Las dos visiones demuestran también las evidentes interacciones especie-de-planta $x$ especie-deanimal, que se comprueba claramente en esta Tabla $1(I x I I, \mathrm{P}<0.001)$ y el efecto individual de la especie de forraje $(I, P<0.001)$, lo que implica la necesidad de diferenciar los cortes que se realicen a cada follaje según sea el animal por alimentar.

Otra implicación importante de este resultado es la necesidad de hacer precisos ajustes individuales en la metodología de evaluación nutritiva de estos follajes 
donde se usan ovinos como especie patrón y que sus resultados sean extrapolados a los bovinos y caprinos, a sabiendas de que muchas variables son estimadas a partir de los datos obtenidos con la especie patrón (Cáceres \& González, 2000).

Muy notable es el resultado de que los ovinos no consumen ninguna porción de los tallos de GS y SS, sino que simplemente se limitan a ingerir hasta los peciolos de las hojas. En exposiciones acerca de GS se han apreciado bajas aceptabilidades cuando se ofrece fresca, probablemente por su olor (Lowry 1990) y también acerca de la aceptabilidad de SS, Delgado et al. (2014) indican que su follaje es poco preferido. En ambos casos, lo destacable es que los bovinos sí consumen hasta dimensiones significativas de los tallos, pero los ovinos no, por tanto, todo está más ligado a algún factor dependiente del animal.

No existen efectos de los dos grupos etarios de animales $(P=0.579)$ para ninguna de las especies de plantas evaluadas, lo que sugiere que puede ser similar el corte que se le haga a los animales jóvenes y adultos, independientemente de cuál es la especie de forraje que se le suministre.

Tabla 1. Efectos de la especie de forraje en el diámetro máximo de los tallos de cinco forrajes de arbustivas consumidas por bovinos y ovinos jóvenes o adultos.

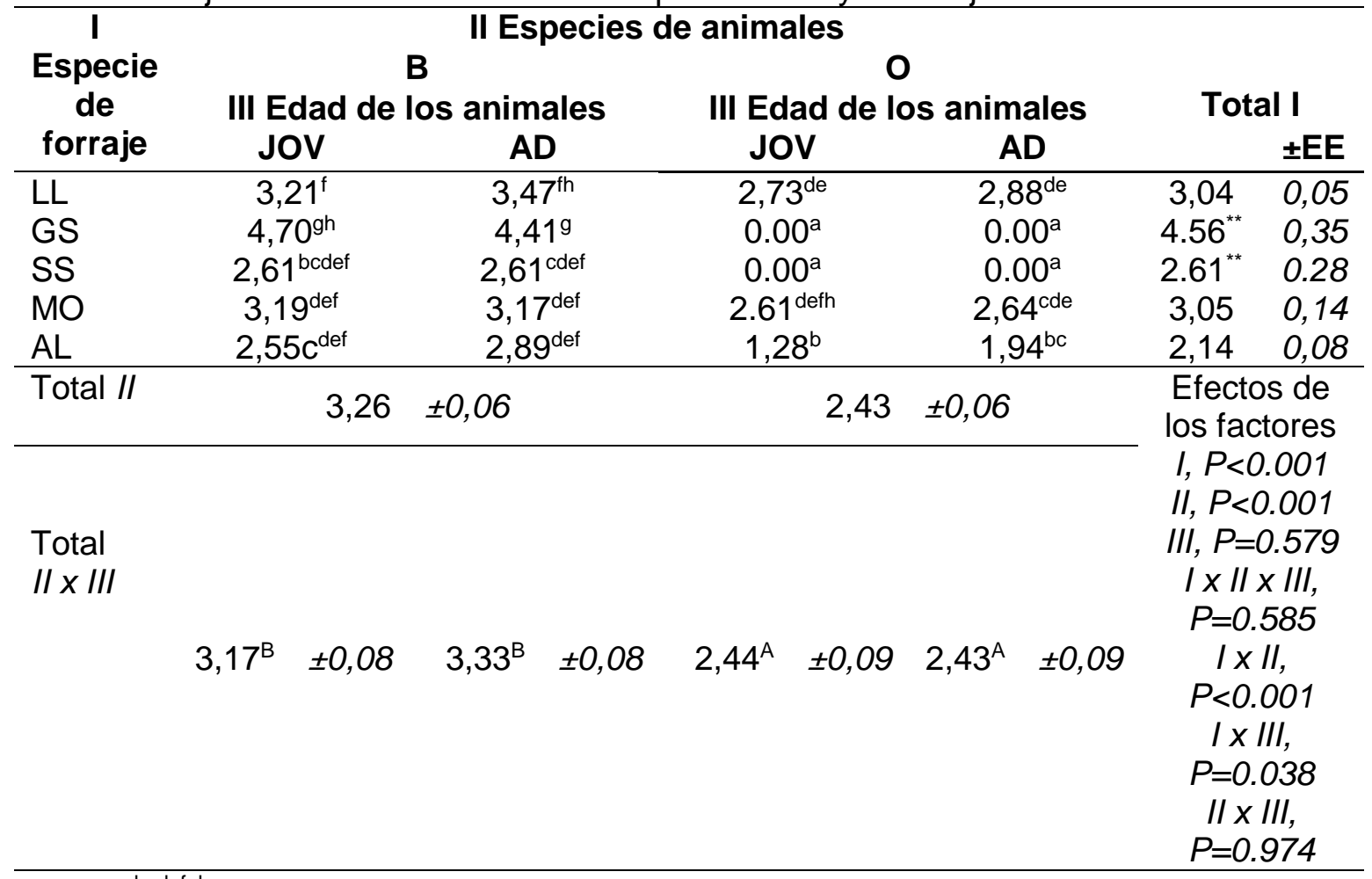

abcdefgh Medias con superíndices en minúsculas iguales implican diferencias no significativas para $\mathrm{P}<0.05$ según la prueba de Tukey.

$\mathrm{AB}$ Medias con superíndices en mayúsculas iguales en la misma línea implican diferencias no significativas para $\mathrm{P}<0.05$ según la prueba de Tukey.

Factores: I Especie del forraje, II Especie de animal, III Edad del animal. EE Error Estándar

${ }^{*}$ Solamente incluye los resultados de los bovinos. 
En la Tabla 2 se pueden observar algunos aspectos que justifican en gran medida el diámetro hasta el cual son consumidos los tallos de las especies evaluadas. El mayor CV de las medias cuantificadas en MO es indicativo de una gran dispersión de los diámetros medidos tanto en los bovinos como en los ovinos, y esto implica que otros factores, además de los evaluados, podrían haber influido. También lo justifica el hecho de que con esta especie hubo que dedicar siete días de adaptación de los animales al follaje para que se notara un consumo ávido por estos. Con SS fue similar el proceso para los bovinos, adicionalmente se destaca que los ovinos no consumieron porción alguna de los tallos.

Tabla 2. Estadígrafos de los diámetros de los tallos consumidos de las cinco especies por bovinos y ovinos de dos edades.

\begin{tabular}{|l|c|c|c|c|c|c|}
\hline Tratamientos & $\mathbf{N}$ & Diámetro medio & Mín. & Máx. & DE & CV \\
\hline LL-B-JOV & 391 & 3,215 & 1,904 & 5,262 & 0,750 & 23,3 \\
\hline LL-B-AD & 494 & 3,475 & 1,318 & 6,260 & 1,020 & 29,4 \\
\hline LL-O-AD & 409 & 2,877 & 1,158 & 5,620 & 0,962 & 33,4 \\
\hline LL-O-JOV & 520 & 2,729 & 1,173 & 5,415 & 0,815 & 29,9 \\
\hline GS-B-AD & 170 & 4,410 & 3,128 & 5,983 & 0,639 & 14,5 \\
\hline GS-B-JOV & 260 & 4,697 & 4,128 & 5,454 & 0,597 & 12,7 \\
\hline MO-B-AD & 285 & 3,170 & 1,513 & 6,138 & 1,238 & 39,1 \\
\hline MO-B-JOV & 249 & 3,192 & 1,440 & 6,040 & 1,276 & 40,0 \\
\hline MO-O-AD & 125 & 2,644 & 1,414 & 7,456 & 1,814 & 68,6 \\
\hline MO-O-JOV & 120 & 2,613 & 1,225 & 5,838 & 1,412 & 54,0 \\
\hline AL-B-AD & 380 & 2,891 & 1,391 & 5,509 & 1,074 & 37,1 \\
\hline AL-B-JOV & 189 & 2,547 & 1,360 & 4,478 & 1,088 & 42,7 \\
\hline AL-O-AD & 262 & 1,938 & 0,960 & 3,824 & 0,711 & 36,7 \\
\hline AL-O-JOV & 224 & 1,278 & 0,858 & 1,746 & 0,258 & 20,2 \\
\hline SS-B-AD & 169 & 2,606 & 1,230 & 5,205 & 1,288 & 49,4 \\
\hline SS-B-JOV & 263 & 2,609 & 1,583 & 4,862 & 1,049 & 40,2 \\
\hline
\end{tabular}

Para GS queda claro que al poseer un bajo valor del CV para los bovinos y no ser consumida por los ovinos, los factores determinantes son propios de la interacción planta-animal. De la misma manera con LL, que posee aceptables valores del CV y los animales solo necesitaron dos días para aceptarla. Se demuestra que es preferida por los animales y consumida homogéneamente tanto por los bovinos como por los ovinos.

En condiciones de pastoreo, el amplio rango de arquitectura de las plantas arbóreas y su diversidad de estratos influyen en el consumo por los animales (White \& Hood, 2004; Flores, Vermont-Ricalde \& Kantún-Balam, 2006; González-Pech et al., 2014) porque determinan el tamaño y frecuencia del bocado. Pero en la presente investigación, por estar los animales estabulados al momento de la suplementación con los alimentos ofrecidos en los comederos y por las semejanzas morfológicas de las hojas de las plantas, las diferencias entre los consumos están más determinados por su palatabilidad.

Algunos autores han medido el aprovechamiento que se hace durante el ramoneo de las especies arbustivas, luego de haber observado, medido y marcado diferentes 
ramas, del pastoreo y de una segunda medición (Clark, 2000; Avendaño et al., 2003; Pontes et al., 2010; González-Pech et al., 2014) y han demostrado las interrelaciones entre las características fenológicas de las plantas y el consumo de los animales. La Figura 1 muestra las marcadas diferencias morfológicas entre los tallos de las cinco especies evaluadas, ya que inicialmente el intercepto de cada ecuación es diferente: mientras los tallos de GS apenas a $1 \mathrm{~cm}$ de su parte más apical ya miden casi $4 \mathrm{~mm}$ de diámetro, LL posee solamente $2.5 \mathrm{~mm}$ de diámetro; luego, la tasa de engrosamiento de los tallos, según avanza su longitud, es muy diferente y explica que a una longitud de $35 \mathrm{~cm}$ los tallos de GS sobrepasen los 9 $\mathrm{mm}$ de diámetro; en cambio, LL solo alcanza unos $4 \mathrm{~mm}$ a esa longitud.

Los valores más bajos en el coeficiente $R$ de las ecuaciones para SS y Al pueden ser explicados porque estas dos especies poseen tallos con cortezas muchos más irregulares, mientras que LL, GS y MO tienen tallos más lisos, lo que hace más homogéneos los datos de su diámetro. Esto implica que es mucho más fácil de medir el diámetro en estas tres últimas especies y que aumenta el valor predictivo de la longitud del tallo para decidir dónde hacer los cortes. También se sabe que la presencia de callosidades en los tallos de las plantas reduce a corto plazo su consumo por los animales (Laca et al., 2001) porque pueden dañar los tejidos suaves del morro, los labios, ojos, piel y hasta las orejas de estos últimos, aunque a largo plazo desarrollan estrategias para incrementar el consumo (Provenza \& Ropp, 2001; Papachristou et al., 2005).

Teniendo en cuenta que considerar la longitud de las ramas que han de ser cortadas, en lugar de su diámetro, es mucho más práctico, esta Figura 1 también permite estimar y recomendar hasta qué longitud deben ser cortados los tallos de cada una de las especies para lograr un diámetro del tallo que sí va a ser consumido por los animales y que asegure un máximo de $5 \%$ de pérdidas (o $95 \%$ de aprovechamiento) en la biomasa cortada (Tabla 3), lo que es de gran importancia para los productores que deben decidir en qué estado debe ser pastoreada 0 cortada la planta, para quienes formulan raciones y para quienes toman muestras para ser enviadas a los laboratorios de análisis químicos.

Como se aprecia, las ramas secundarias y terciarias de Leucaena pueden ser cortadas hasta $46 \mathrm{~cm}$ de longitud, en cambio las de GS y SS deben ser defoliadas totalmente cuando vayan a ser ofrecidas a los ovinos, porque estos no consumen sus tallos.

Los ovinos y caprinos que ramonean en un bosque caducifolio de Yucatán, en México, consumen tallos de diferentes arbustos hasta unos $20 \mathrm{~cm}$ de longitud (González-Pech et al., 2014), lo que se puede considerar como similar al recomendado para los ovinos en el corte de la Leucaena, como valor máximo entre todas las especies evaluadas. Pero, sin duda, es necesario hacer las diferenciaciones necesarias para cada especie de forraje y de animal, por las significativas interacciones encontradas en los resultados de la Tabla 1. 


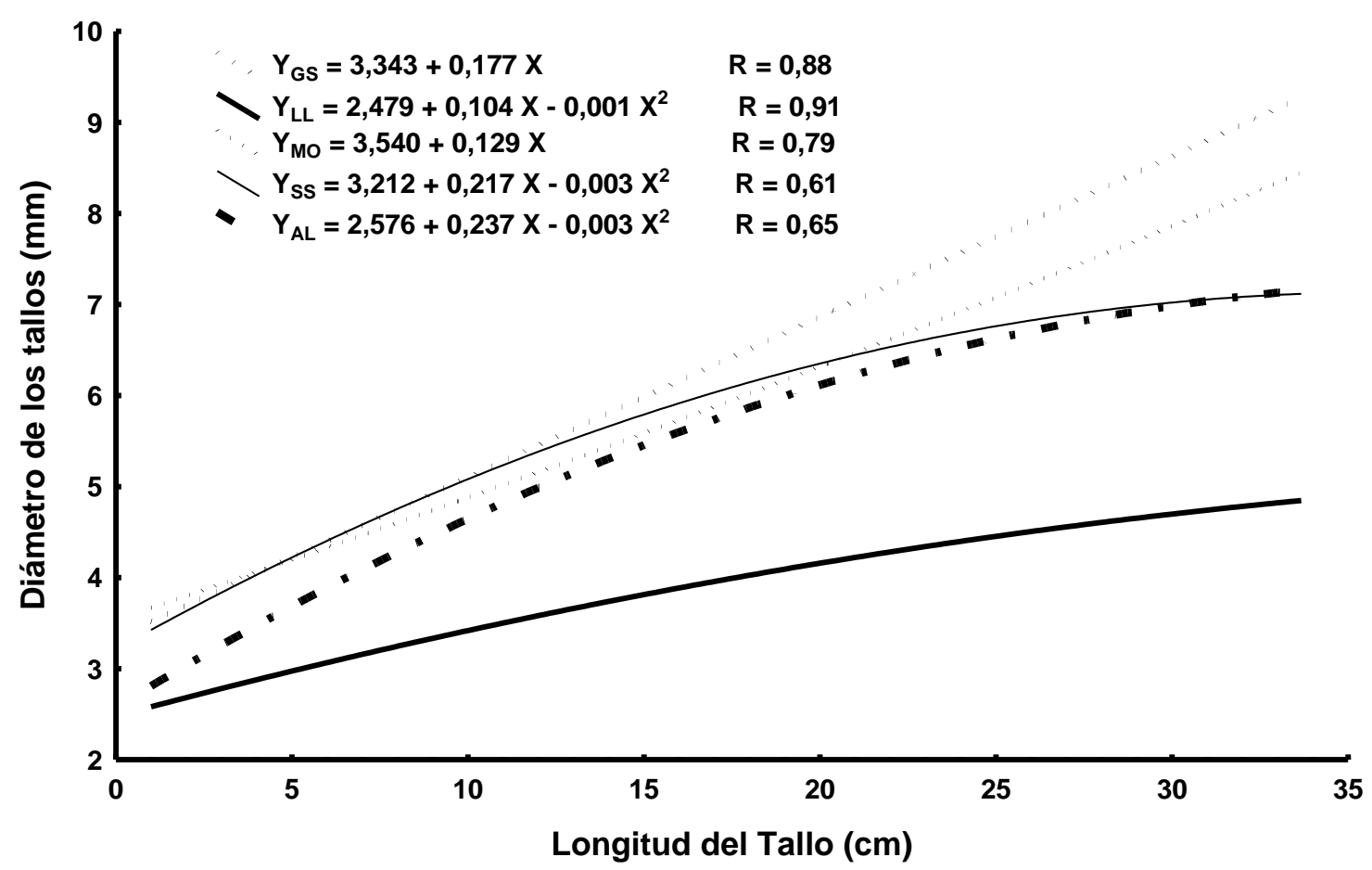

Fig. 1. Relación entre la longitud de los tallos y su diámetro en cinco especies de arbustivas (GS, Gliricidia sepium; LL, Leucaena leucocephala; MO, Moringa oleifera; SS, Samanea saman; AL, Albizia lebbeck).

Tabla 3. Diámetro máximo y longitud de los tallos para cortar las cinco especies evaluadas.

\begin{tabular}{|l|c|c|c|c|}
\hline \multirow{2}{*}{\multicolumn{1}{|c|}{ Especie }} & \multicolumn{2}{c|}{ Diámetro (mm) } & \multicolumn{2}{c|}{ Longitud (cm) } \\
\cline { 2 - 5 } & Bovinos & Ovinos & Bovinos & Ovinos \\
\hline Leucaena leucocephala & 5 & 4 & 46 & 26 \\
\hline Gliricidia sepium & 6 & 0 & 13 & 0 \\
\hline Samanea saman & 5 & 0 & 7 & 0 \\
\hline Moringa oleifera & 5 & 5 & 14 & 15 \\
\hline Albizia lebbeck & 5 & 3 & 9 & 2 \\
\hline
\end{tabular}

\section{CONCLUSIONES}

Los resultados obtenidos en las pruebas con los animales y las estimaciones hechas a partir de estos, demuestran que no deben ser cortadas por igual todas las especies arbustivas evaluadas, puesto que existen grandes diferencias entre ellas. Tampoco debe ser igual cuando se trata de alimentar bovinos u ovinos, porque ambas especies no aprovechan de la misma manera las diferentes plantas. En cambio, se puede suministrar cada follaje por igual a animales en desarrollo y a animales adultos, pues el aprovechamiento es similar entre ellos. Se aconseja que los cortes a estas plantas arbustivas-para alimentar a los animales o en investigaciones - sean hechos teniendo en cuenta las dimensiones propuestas en la Tabla 3; que se realicen pruebas similares sobre el aprovechamiento de otras especies de plantas y que se emplee la metodología de medir el diámetro de los 


\section{tallos rechazados por los animales y correlacionarlo con la longitud de las ramas para recomendar su corte con base en estas dos dimensiones.}

\section{REFERENCIAS}

ACPA-SOCUPER (2002). Ovino Pelibuey: Manual. Cuba: Asociación Cubana de Producción Animal.

Agreil, C., Fritz, H., \& Meuret, M. (2005). Maintenance of Daily Intake through Bite Mass Diversity Adjustment in Sheep Grazing on Heterogeneous and Variable Vegetation. Applied Animal Behaviour Science, 91(1-2), 35-56. https://doi.org/10.1016/j.applanim.2004.08.029.

Aregheore, E. M. (2003). Nutritional Characterization and Evaluation of Batiki Grass (Ischaemum aristatum var. indicum) and Batiki Grass-Legume Mixtures with Steer Calves. Journal of Animal and Veterinary Advances, 2(7), 404-412. https://doi.org/10.1016/s0921-4488(03)00198-6.

Avendaño, J., Ovalle, C. M., \& Ramírez, M. (2003). Caracterización de los componentes vegetales consuidos por ovinos y bovinos en plantas de Tagasaste (Chamaecytisus proliferus ssp. palmensis). Agricultura Técnica, 63(1), 38-47. https://doi.org/10.4067/s0365-28072003000100006.

Bonnet, O. J. F., Meuret, M., Tischler, M. R., Cezimbra, I. M., Azambuja, J. C. R., \& Carvalho, P. C. F. (2015). Continuous Bite Monitoring: A Method to Assess the Foraging Dynamics of Herbivores in Natural Grazing Conditions. Animal Production Science, 55(3), 339-349. https://doi.org/10.1071/AN14540.

Cáceres, O., \& González, E. (2000). Metodología para la determinación del valor nutritivo de los forrajes tropicales. Pastos y Forrajes, 23(2), 87-103.

Clark, A., 2000. Controlling broom (Cytisus scoparius) in pasture on the Barrington Tops-a graziers perspective. Plant Protection Quarterly, 15, 161-162.

Delgado, D.C., Hera, R., Cairo, J. \& Orta, Y. (2014). Samanea saman, a multi-purpose tree with potentialities as alternative feed for animals of productive interest. Cuban Journal of Agricultural Science, 48(3), $205-212$.

Dziba, L. E., Scogings, P. F., Gordon, I. J., \& Raats, J. G. (2003). Effects of Season and Breed on Browse Species Intake Rates and Diet Selection by Goats in the False Thornveld of the Eastern Cape, South Africa. Small Ruminant Research, 47(1), 17-30. https://doi.org/10.1016/S0921-4488(02)00235-3.

FAO-UNESCO (1988). Soil Map of the World. Revised Legend. World Soil Resources. Rome, Italy: FAOUNESCO.

Fisher, D. S. (2002). A Review of a Few Key Factors Regulating Voluntary Feed Intake in Ruminants. Crop Science, 42(5), 1651-1655. https://doi.org/10.2135/cropsci2002.1651.

Flores, G., Amor J., Resch, C., \& González, A. (2000). Valor nutritivo del ensilaje de hierba en las explotaciones ganaderas de Galicia. Pastos, 30(2), 149-191.

Flores, S. J., Vermont-Ricalde, M. R., \& Kantún-Balam, M. J. (2006). Leguminosae Diversity in the Yucatan Peninsula and its Importance for Sheep and Goats Feeding. In C.A. Sandoval-Castro, F.D. Hovel, J.F.J. Torres-Acosta \& A. Ayala-Burgos (eds.). Herbivores: The Assessment of Intake, Digestibility and the Roles of Secondary Compounds. 291-299. Nottingham, United Kingdom: Nottingham University Press.

Forbes J. M. (2000). Physiological and Metabolic Aspects of Feed Intake Control. In J.P.F. D’Mello (ed), Farm Animal Metabolism Nutrition. United Kingdom: CAB International. https://doi.org/10.1079/9780851993782.0319.

Funes, F., \& Jordán, H. (1987). Leche. En T. Ruiz (Ed.). Leucaena, una opción para la alimentación bovina en el trópico y el subtrópico. Ed. Instituto de Ciencia Animal. Cuba: 129 p.

García, D. E., Medina, M. G., \& Ojeda, F. (2005). Carbohidratos solubles en cuatro variedades de morera (Morus alba Linn.). Pastos y Forrajes, 28(3), 233-239.

Ciencia y Agricultura (Cien. Agri.) Vol. 16 (2). L-ISSN 0122-8420. elSSN 2539-0899.

Mayo-Agosto 2019, pp. 25-38. Tunja (Boyacá) - Colombia. DOI:

https://doi.org/10.19053/01228420.v16.n2.2019.9118 
García, R. \& O. Cáceres. (1984). Nuevos sistemas para expresar el valor nutritivo de los alimentos y el requerimiento y racionamiento de los rumiantes. EEPF "Indio Hatuey", Matanzas. Cuba. 44 p.

Geisert, B. G. (2007). Development of a Set of Forage Standard to Estimate in vivo Digestibility of Forages and Prediction of Forage Quality of Diets Consumed by Cattle Grazing Nebraska Sandhills Range Pastures. (Tesis de Doctorado). University of Nebraska, Lincoln. USA. Retrieved from http://digitalcommons.unl.edu/animalscidiss/2.

González-García, E., Cáceres, O., Archimède, H., \& Santana, H. (2009). Nutritive Value of Edible Forage from Two Leucaena leucocephala Cultivars with Different Growth Habit and Morphology. Agroforestry Systems, 77(2), 131-141. http://doi.org/10.1007/s10457-008-9188-4.

González-Pech, P. G., Torres-Acosta, J. F., \& Sandoval-Castro, J. (2014). Adapting a Bite Coding Grid for Small Ruminants Browsing a Deciduous Tropical Forest. Tropical and Subtropical Agroecosystems, 17, 63-70.

Gutiérrez, O., Delgado, D., Oramas, A., \& Cairo, J. (2005). Consumo y digestibilidad de materia seca y nitrógeno total en vacas en pastoreo durante la época de lluvias, con bancos de proteína y sin ellos. Revista Cubana de Ciencia Agrícola, 39(4), 593-597. https://doi.org/10.24188/recia.v7.n2.2015.264.

Hernández, I., \& Sánchez, M. D. (2014). Small Ruminant Management and Feeding with High Quality Forages in the Caribbean. Santo Domingo, República Dominicana: IICA.

Iglesias, J. M., Matías, C., \& Pérez, A. (2003). Cría de hembras bovinas en desarrollo en condiciones de silvopastoreo. Pastos y Forrajes, 26(1), 35-46.

Ingram, R.S., Doran, M.P. \& Nader, G. (2013). Planned Herbivory in the Management of Wildfire Fuels In B. Barros \& M.E.B. Fernandes (eds.), Herbivory InTech. 61-86. Rijeka. Croacia. https://doi.org/10.5772/2718.

Kapinga, P. X., Bitende, S. N., \& Mulangila, R. C. T. (1993). Evaluation of Sesbania sesban and Sesbania goetzei Accessions in Subhumid Coastal Area of Tanga, Tanzania. En J. Ndikumana \& P. de-Leeuw (eds), Proceedings of the Second African Feed Resources Network, 46-51. Zimbabwe: Harare.

Karsli, M. A., \& Russell, J. R. (2002). Prediction of the Voluntary Intake and Digestibility of Forage-Based Diets from Chemical Composition and Ruminal Degradation Characteristics. Turkish Journal of Veterinary and Animal Science, 26, 249-255.

Laca, E. A., Shipley, L. A., \& Reid, E. D. (2001). Structural Anti-Quality Characteristics of Range and Pasture Plants. Journal of Range Management, 54(4), 413-419. https://doi.org/10.2307/4003112.

Lowry, J. B. (1990). Toxic Factors and Problems: Methods of Alleviating them in Animals. En: C. Devendra (ed.), Proceedings of a workshop in Denpasar, 76-91. Indonesia.

Marais, J. P. (2000). Use of Markers. En J.P.F. D’Mello (ed.), Farm Animal Metabolism Nutrition, 255-277. United Kingdom: CAB International. https://doi.org/10.1079/9780851993782.0255.

NRC (2005). Mathematics and 21st Century Biology. Committee on Mathematical Sciences Research for DOE's Computational Biology, National Research Council. Recuperado de: http://www.nap.edu/catalog/11315.html.

Mathews, G. L., \& Ellinbank, G. A. C. (1989). Utilization of Tagasaste. Tagasaste, Research Results and Farmer Experiences. Small Ruminant Research, 59(2-3), 141-156. https://doi.org/10.1016/j.smallrumres.2005.05.003.

Parissi, Z. M., \& Nastis, A. S. (2007). Nutritive Value of White Oak (Quercus pubescens Wild.) Browsed by Goats. In A. Priolo, L. Biondi, H. Ben Salem, H. \& P. Morand-Fehr (eds.), Advanced Nutrition and Feeding Strategies to Improve Sheep and Goat, 193-196. Zaragoza: CIHEAM.

Paterson, R. T., Quiroga, L., Sauma, G., \& Samur, C. (1983). Crecimiento de novillas Cebú-Criollo en la época de seca con acceso limitado a la Leucaena. Producción Animal, 8, 150-162. 
Pontes, L. S., Agreil, C., Magda, D., Gleizes, B., \& Fritz, H. (2010). Feeding Behavior of Sheep on Shrubs in Response to Contrasting Herbaceous Cover in Rangelands Dominated by Cytisus scoparius L. Applied Animal Behaviour Science, 124(1-2), 35-44. https://doi.org/10.1016/j.applanim.2010.02.002.

Provenza, F. D., \& Ropp, J. (2001). Understanding Herbivore Response to anti-Quality Factors in Forages. In K. Launchbaugh (ed.), Antiquality Factors in Rangeland and Pastureland Forages, 5-12. USDA: Idaho Forest, Wildlife and Range Experiment Station. https://doi.org/10.2458/azu jrm v54i4 launchbaugh.

Santana, A. A., Pernía, L. A., \& Santana, D. A. (2015). Historical, Mathematical and Nutritional Bases of Pearson Square as a Fit Method for Ruminant Rations. Cuban Journal of Agricultural Science, 49(3), 279-288.

Sebata, A., \& Ndlovu, L. R. (2010). Effect of Leaf Size, Thorn Density and Leaf Accessibility on Instantaneous Intake Rates of Five Woody Species Browsed by Matebele Goats (Capra hircus L) in a Semi-Arid Savanna, Zimbabwe. Journal of Arid Environment, 74(10), 1281-1286. https://doi.org/10.1016/j.jaridenv.2010.05.028.

Sebata, A. 2013. Woody Plant-Herbivore Interactions in Semi-Arid Savanna Ecosystems. En B. Barros \& M.E.B. Fernandes (eds.), Herbivory InTech, 1-17. Rijeka. Croacia. https://doi.org/10.5772/2718.

Valenciaga, D. \& Chongo, B. (2004). La pared celular. Influencia de su naturaleza en la degradación microbiana ruminal de los forrajes. Revista Cubana de Ciencia Agrícola, 38(4), 343-350.

White, D. A., \& Hood, C. S. (2004). Vegetation Patterns and Environmental Gradients in Tropical Dry Forest of the Northern Yucatan Peninsula. Journal Vegetation Science, 15(2), 151-160. https://doi.org/10.1111/j.1654-1103.2004.tb02250.x.

Wilson, S. L., \& Kerley, G. I. H. (2003). Bite Diameter Selection by Thicket Browsers: The Effect of Body Size and Plant Morphology on Forage Intake and Quality. Forest Ecology and Management, 181(1-2), 51-65. https://doi.org/10.1016/S0378-1127(03)00114-2.

Ciencia y Agricultura (Cien. Agri.) Vol. 16 (2). L-ISSN 0122-8420. elSSN 2539-0899.

Mayo-Agosto 2019, pp. 25-38. Tunja (Boyacá) - Colombia. DOI:

https://doi.org/10.19053/01228420.v16.n2.2019.9118 